\title{
Viscosity from elliptic flow: Clearing the path towards precision
}

\author{
Ulrich Heinz, John Scott Moreland, and Huichao Song \\ Department of Physics, The Ohio State University, Columbus, Ohio 43210-1117, USA
}

(Received 18 August 2009; published 8 December 2009)

\begin{abstract}
Using viscous relativistic hydrodynamics we show that systematic studies of the impact parameter dependence of the eccentricity-scaled elliptic flow $v_{2} / \varepsilon$ can distinguish between different models for the calculation of the initial source eccentricity $\varepsilon$. This removes the largest present uncertainty in the extraction of the specific viscosity of the matter created in relativistic heavy-ion collisions from precise elliptic-flow measurements.
\end{abstract}

DOI: 10.1103/PhysRevC.80.061901

PACS number(s): 25.75.Dw, 24.10.Nz, 25.75.Ld

Heavy-ion collisions at the Relativistic Heavy Ion Collider (RHIC) produce strongly interacting matter at extremely high energy densities (a quark-gluon plasma), exhibiting hydrodynamic behavior. In noncentral collisions, the collective flow is anisotropic. The degree of anisotropy, measured by the Fourier coefficients $v_{n}$ of the emitted particle distributions in the plane transverse to the beam, is sensitive to the viscosity of the expanding fireball medium [1-4]; the largest anisotropies correspond to fluids with the least viscosity. Measurements of the elliptic-flow coefficient $v_{2}$ (which, at midrapidity, dominates all other $v_{n}$ ) in $200 \mathrm{~A} \mathrm{GeV} \mathrm{Au}+\mathrm{Au}$ collisions have been compared with viscous relativistic hydrodynamic simulations of the fireball matter, yielding an upper limit for the specific shear viscosity (i.e., the dimensionless ratio between shear viscosity $\eta$ and entropy density $s$ ) of $\frac{\eta}{s}<0.5[5,6]$. This is smaller than the minimal values (typically found near the liquid-gas transition [7]) of the corresponding ratio measured in all other known (real) liquids [8], with the possible exception of strongly interacting systems of ultracold fermionic atoms near the unitarity limit [9]. In this sense, the quark-gluon plasma (QGP) appears to be the most perfect liquid ever observed.

The uncertainty relation places a lower bound on the specific shear viscosity [10], and explicit computation in a large class of strongly coupled field theories, using the anti-deSitter/conformal field theory (AdS/CFT) correspondence, puts it near $\left(\frac{\eta}{s}\right)_{\mathrm{KSS}}=\frac{1}{4 \pi} \approx 0.08$ [8]. The above empirical bound is sufficiently close to this fundamental limit to have generated widespread interest in a precise measurement of the specific shear viscosity of the matter created at the RHIC. This requires a multidimensional analysis of a range of observables and an accurate knowledge of a suite of other ingredients whose influence on the measurements must be separated from $\frac{\eta}{s}$. It thus came as an unwelcome surprise when it was realized that our insufficiently precise knowledge of one of these ingredients, the initial fireball eccentricity $\varepsilon=\frac{\left\langle y^{2}-x^{2}\right\rangle}{\left\langle y^{2}+x^{2}\right\rangle}[11,12]$ (which, through anisotropic pressure gradients, drives the flow anisotropy), introduces a large dominant and apparently irreducible uncertainty into the extraction of the specific shear viscosity that, for $\frac{\eta}{s} \sim \mathcal{O}\left(\frac{1}{4 \pi}\right)$, can be $100 \%$ or more [5].

Let us explain the situation in more detail. Elliptic flow appears to be the observable showing the strongest sensitivity

*heinz@mps.ohio-state.edu to shear viscosity. Two main manifestations of shear viscous effects have been identified: (i) Shear viscosity reduces the amount of elliptic flow below the value generated in an ideal fluid [1-4]; for fixed initial conditions, in particular for a given initial fireball eccentricity, the "viscous suppression factor" grows monotonically with $\eta / s$ [3-5]. (ii) When scaled by the initial eccentricity, the elliptic flow for fixed $\eta / s$ is reduced more strongly in smaller collision systems that create shorterlived fireballs than for larger, longer-lived fireballs. Viscous effects are stronger in peripheral than in central collisions, larger in $\mathrm{Cu}+\mathrm{Cu}$ than in $\mathrm{Au}+\mathrm{Au}$ collisions, and become weaker at higher collision energies [13,14].

Based on observation (i), Luzum and Romatschke [5] made a first attempt to extract $\eta / s$ at midrapidity from experimental elliptic flow measurements in minimum bias $\mathrm{Au}+\mathrm{Au}$ collisions at $\sqrt{s}=200 \mathrm{~A} \mathrm{GeV}$. A key ingredient of such an analysis is the ideal fluid dynamical baseline with which the data are compared to establish the "viscous suppression factor" that is used to extract $\eta / s$. In ideal fluid dynamics the elliptic flow is directly proportional to the initial fireball eccentricity, but the latter cannot be experimentally accessed because there are no known probes of the reaction zone that escape directly from the fireball and probe only the initial state, without any contributions from later stages of the expansion. It must therefore be estimated theoretically from the overlap geometry corresponding to the impact parameter of the collision that can be extracted from measurements of the final hadron multiplicity and transverse energy. Unfortunately, theoretical models used to calculate the initial energy and entropy density distributions for given impact parameters differ by up to $30 \%$ in the predicted source eccentricity $[11,12]$. In the study in Ref. [5], two models were studied whose eccentricities $\varepsilon$ differed by about $20 \%$. The corresponding $20 \%$ variation in the ideal fluid baseline for the elliptic flow $v_{2}$ led to variations of more than a factor of 2 in the extracted value of $\eta / s$. While the hydrodynamical calculations in Ref. [5] made several other assumptions (in particular in the equation of state) that affect the ideal fluid baseline for $v_{2}$, their combined effects are likely smaller than that produced by the uncertainty in $\varepsilon$. More importantly, however, it is known how to systematically improve on these approximations in the future and thus dramatically reduce their contribution to the systematic error of $\eta / s$. However, it appears impossible to eliminate the uncertainty in the source eccentricity on purely theoretical grounds, and it is not known whether it can ever be 
measured experimentally. With the method used in Ref. [5], a precise meaurement of $\eta / s$, with errors below about a factor of 2, thus appears to be impossible.

In this article we exploit observation (ii) and identify measurements that will clearly distinguish between the two models for $\varepsilon$ studied in Ref. [5]. This clears a possibly terminal roadblock from the path toward a precise extraction of the QGP bulk and shear viscosities [6,15]. We stress that in the work presented here we do not follow this path to its end; for a precise extraction of $\eta / s$ from experimental data, other sources of uncertainty must be eliminated, as discussed above. This can be done with systematic theoretical improvements that are technically involved (and hence go beyond the scope of this short article) but for which, in principle, the solution is known. The point of the work presented here is to show how the one source of (large!) uncertainty for which no theoretical solution is known can be eliminated phenomenologically, by studying systematic trends of the eccentricity-scaled elliptic flow $v_{2} / \varepsilon$ as a function of system size and collision centrality.

Figure 1 shows the initial spatial fireball eccentricity calculated from the Glauber [16] and factorized KharzeevLevin-Nardi (fKLN) [12,17] models for $200 A \mathrm{GeV} \mathrm{Au}+\mathrm{Au}$ collisions as a function of impact parameter $b$. It is defined as

$$
\varepsilon(b)=\frac{\int d^{2} x_{\perp}\left(y^{2}-x^{2}\right) e\left(\boldsymbol{x}_{\perp} ; b\right)}{\int d^{2} x_{\perp}\left(y^{2}+x^{2}\right) e\left(\boldsymbol{x}_{\perp} ; b\right)},
$$

where $e(x, y ; b)$ is the energy density in the transverse plane at $z=0$; here $z$ denotes the beam direction, $x$ the direction of the impact parameter, and $y$ points orthogonal to the reaction plane. For the Glauber model, $e\left(\boldsymbol{x}_{\perp} ; b\right)$ is taken to be proportional to a superposition of wounded nucleon $(85 \%)$ and binary collision (15\%) densities [11]. In the fKLN model, the shape of $e\left(x_{\perp} ; b\right)$ is controlled by the dependence of the gluon saturation momentum $Q_{s}$ on the transverse position $\boldsymbol{x}_{\perp}$. We compute it according to Ref. [12], using a program kindly provided by the authors [18].

A quick look at Fig. 1 shows that eccentricities from the fKLN model are $\mathcal{O}(20 \%-30 \%)$ larger than those from the Glauber model $[11,12]$. Closer inspection reveals that the excess depends strongly on impact parameter (see Fig. 1 inset): At small $b$ the $\varepsilon_{\text {fKLN }}$ is more than $60 \%$ larger than $\varepsilon_{\mathrm{G}}$, whereas at large $b>8 \mathrm{fm}$ the excess drops to $<20 \%$. Had $\varepsilon_{\mathrm{G}}$ turned out to be simply proportional to $\varepsilon_{\mathrm{fKLN}}$, the proportionality constant (and with it the model dependence of the initial eccentricity) could have been eliminated simply by forming the double ratio $\left(v_{2} / \varepsilon\right)_{\text {peripheral }} /\left(v_{2} / \varepsilon\right)_{\text {central }}$ and exploiting the system size dependence of viscous effects to extract $\eta / s$. The inset in Fig. 1 shows that this will not work.

To make progress, let us next look at the sensitivity of the eccentricity-scaled elliptic flow $v_{2} / \varepsilon$ to $\eta / s$ and then proceed to find a way to determine which model for $\varepsilon$ should be used for scaling the experimentally measured elliptic flow. The following results are obtained from viscous hydrodynamic simulations of $200 \mathrm{~A} \mathrm{GeV} \mathrm{Au}+\mathrm{Au}$ collisions, with constant $\eta / s$ and standard initial and final conditions [4,13]. We comment on possible effects from a temperature dependence of $\eta / s$ at the end.

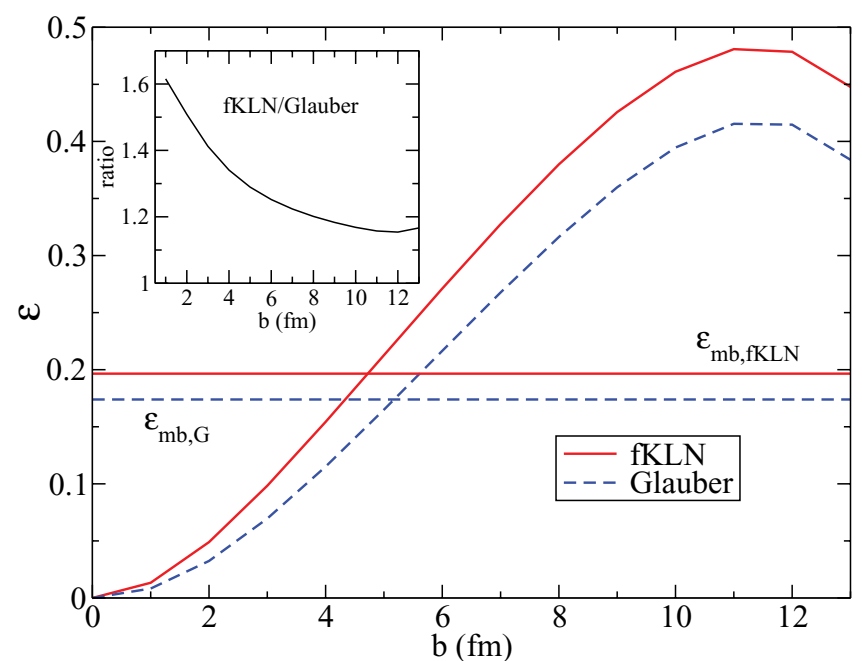

FIG. 1. (Color online) Impact parameter dependence of the initial fireball eccentricities in the Glauber and fKLN models and their ratio (inset). The horizontal lines indicate the corresponding values in minimum bias (i.e., $b$-averaged) events.

In Fig. 2 we show $v_{2}^{\mathrm{mb}} / \varepsilon_{\mathrm{mb}}$ for minimum bias collisions, as a function of $\eta / s . v_{2}^{\mathrm{mb}}$ is obtained from the minimum bias pion spectrum $d N_{\pi}^{\mathrm{mb}} /\left(d y d^{2} p_{T}\right)$ (without resonance decay feeddown), and $\varepsilon_{\mathrm{mb}}$ is computed as above from the minimum bias energy density $e_{\mathrm{mb}}\left(\boldsymbol{x}_{\perp}\right)=2 \int_{0}^{b_{\max }} d b b e\left(\boldsymbol{x}_{\perp} ; b\right) / b_{\max }^{2}$, with $b_{\max }=13 \mathrm{fm}$.

The figure shows that for minimum bias collisions eccentricity scaling works in viscous hydrodynamics, that is, one obtains almost identical curves for different initial eccentricity models (the same does not hold at fixed impact parameters; see below). For any given viscosity $\eta / s$, the scaled elliptic flow depends only on the stiffness on the equation of state: EOS I,

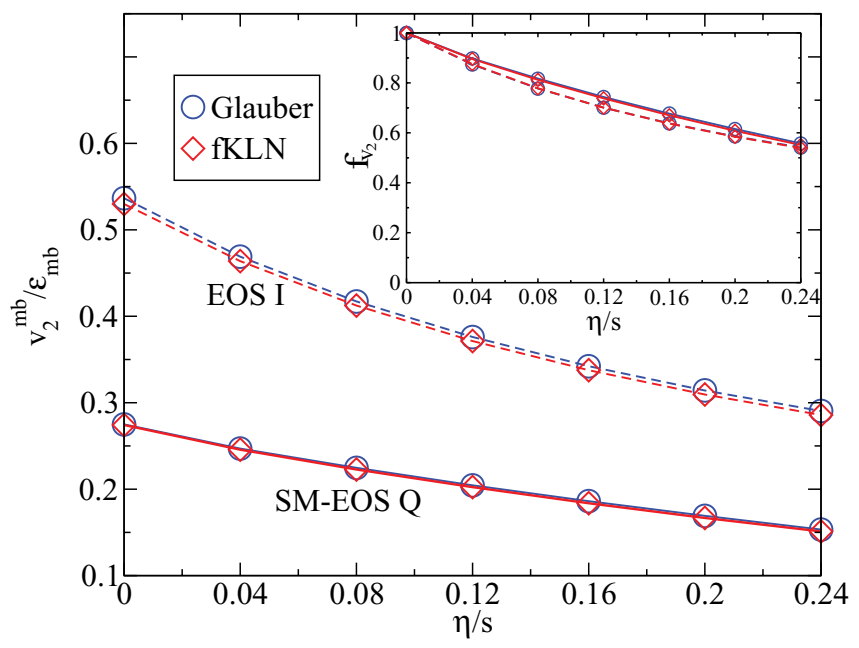

FIG. 2. (Color online) Scaled elliptic flow $v_{2} / \varepsilon$ for minimum bias $200 A \mathrm{GeV} \mathrm{Au}+\mathrm{Au}$ collisions, as a function of specific entropy $\eta / s$, from viscous hydrodynamics with two different equations of state, for the Glauber and fKLN initial state models. (Inset) The fractional viscous suppression of $v_{2}^{\mathrm{mb}} / \varepsilon_{\mathrm{mb}}$ as a function of $\eta / s$. See text for discussion. 
which describes a massless parton gas with $e=3 p$ and sound speed $c_{s}=1 / \sqrt{3}$, gives more elliptic flow per eccentricity than the softer SM-EOS Q [19], which matches a massless parton gas to a hadron resonance gas at $T_{c}=164 \mathrm{MeV}$ through a first-order phase transition, with $c_{s}=0$ in the mixed phase and $c_{s} \approx 1 / \sqrt{6}$ in the hadronic phase. However, the fractional suppression of $v_{2} / \varepsilon$ by shear viscosity below its ideal fluid value is almost independent of the EOS: defining

$$
f_{v_{2}}=\frac{\left(v_{2}^{\mathrm{mb}} / \varepsilon_{\mathrm{mb}}\right)_{\text {viscous }}}{\left(v_{2}^{\mathrm{mb}} / \varepsilon_{\mathrm{mb}}\right)_{\text {ideal }}}
$$

the fraction of scaled elliptic flow generated in viscous hydrodynamics relative to the ideal fluid value, the inset in Fig. 2 shows that this fraction is an approximately universal function of the shear viscosity $\eta / s$, with practically no sensitivity to the initial eccentricity and only weak dependence on the EOS. (The somewhat lower fractions for EOS I are caused by earlier decoupling in this highly explosive case [4], which cuts off the evolution of $v_{2}$ before full saturation [19].) Because the EOS can in principle be obtained from Lattice QCD with arbitrary precision, the EOS dependence is not a concern. What counts is that there is no large dependence on the model for the initial eccentricity $\varepsilon$, which cannot be reliably calculated from first principles.

Figure 2 states that if we know $v_{2}^{\mathrm{mb}} / \varepsilon_{\mathrm{mb}}$ and the EOS, we can determine $\eta / s$. But experimentally one can measure only $v_{2}$; $\varepsilon_{\mathrm{mb}}$ must be calculated from a model. How can we tell which model for $\varepsilon$ is correct? The minimum bias eccentricities for the fKLN and Glauber models $\left(\varepsilon_{\mathrm{mb}}^{\mathrm{fKLN}}=0.197 \mathrm{vs} \varepsilon_{\mathrm{mb}}^{\mathrm{G}}=0.174\right.$; see Fig. 1) differ by $12 \%$, and Fig. 2 shows that the corresponding $\sim 12 \%$ uncertainty in $v_{2} / \varepsilon$ leads to a factor of $\sim 2$ uncertainty in $\eta / s$ when $\eta / s=\mathcal{O}(1 / 4 \pi)$.

The solution to this conundrum is given in Figs. 3 and 4 which, instead of the minimum bias ratios, explore the impact parameter dependence of $v_{2} / \varepsilon$ for EOS I (Fig. 3) and SM-EOS Q (Fig. 4). The left panels in these figures

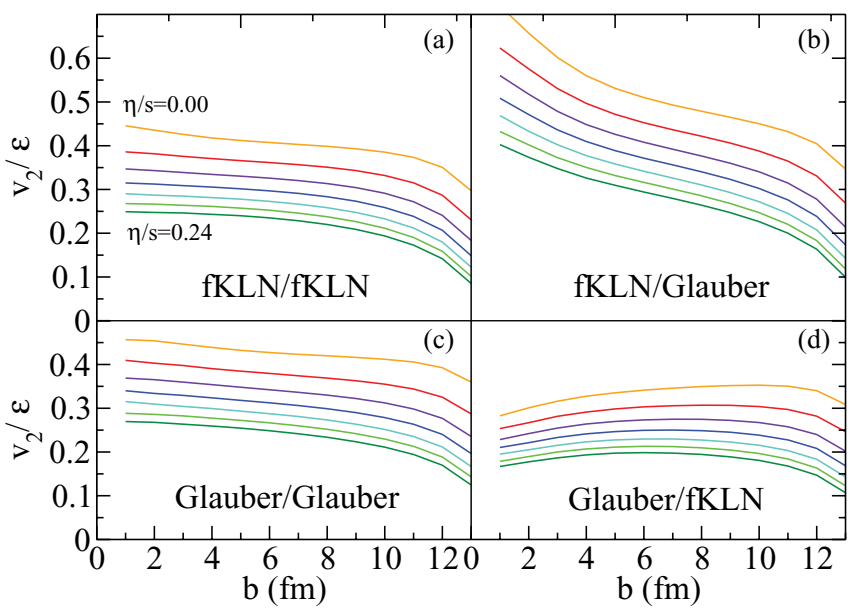

FIG. 3. (Color online) Scaled elliptic flow $\left(v_{2} / \varepsilon\right)(b)$ from viscous hydro with EOS I and either Glauber or fKLN initial conditions. Lines correspond to $\eta / s=0.0,0.04, \ldots, 0.24$ (from top to bottom in steps of 0.04). Panels (a) and (c) show $v_{2}^{\mathrm{fKLN}} / \varepsilon_{\mathrm{fKLN}}$ and $v_{2}^{\mathrm{G}} / \varepsilon_{\mathrm{G}}$, respectively; panels (b) and (d) show the "swapped ratios" $v_{2}^{\mathrm{fKLN}} / \varepsilon_{\mathrm{G}}$ and $v_{2}^{\mathrm{G}} / \varepsilon_{\mathrm{fKLN}}$, respectively.

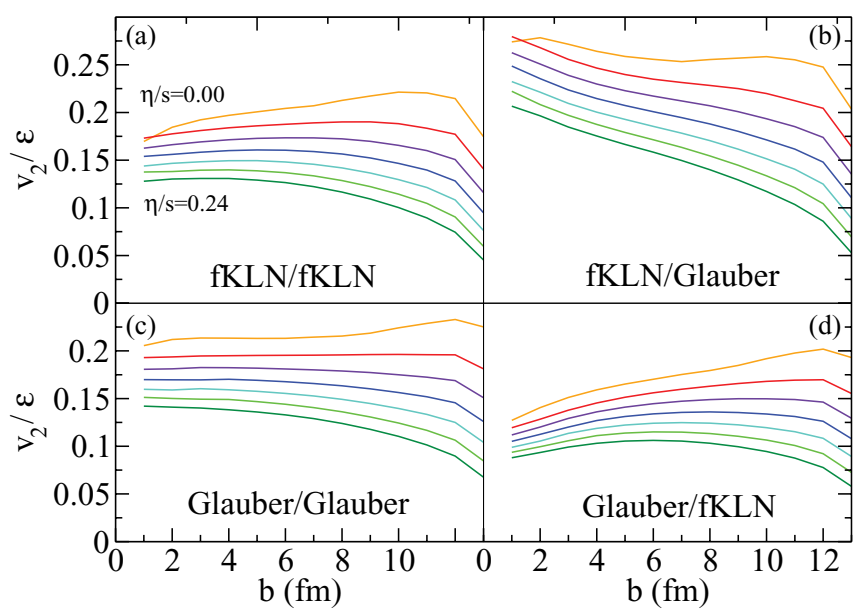

FIG. 4. (Color online) Same as Fig. 3, but for the equation of state SM-EOS Q with a quark-hadron phase transition.

show $v_{2}^{\mathrm{fKLN}}(b) / \varepsilon_{\mathrm{fKLN}}(b)$ (top) and $v_{2}^{\mathrm{G}}(b) / \varepsilon_{\mathrm{G}}(b)$ (bottom). Comparing the top left and bottom left panels in Figs. 3 or 4 one observes approximate eccentricity scaling at all impact parameters, although not with the same degree of precision as for the minimum bias average shown in Fig. 2. At each impact parameter, the viscous suppression of $v_{2} / \varepsilon$ grows monotonically with $\eta / s$.

For EOS I (Fig. 3), $v_{2} / \varepsilon$ decreases monotonically with $b$, reflecting the earlier freezeout and a lower degree of saturation of the elliptic flow in more peripheral collisions. For SM-EOS $\mathrm{Q}$ we observe a more complex pattern: as seen in Figs. 4(a) and $4(\mathrm{c}), v_{2} / \varepsilon$ increases with $b$ for the ideal fluid (except for very large $b$, where early freezeout again takes its toll) but decreases with $b$ for viscous fluids once $\eta / s$ exceeds about once or twice the KSS bound $\left(\frac{\eta}{s}\right)_{\mathrm{KSS}}=\frac{1}{4 \pi}$ (depending on whether we use Glauber or fKLN initial conditions). The increase with $b$ seen for the ideal fluid is well known [19] and reflects the effective stiffening of the EOS (i.e., a larger effective speed of sound) as the system evolves out of the very soft mixed phase (which in central $\mathrm{Au}+\mathrm{Au}$ collisions at top RHIC energies suppresses the buildup of elliptic flow) into the significantly harder hadronic phase (which dominates elliptic-flow buildup in the more peripheral collisions). Shear viscosity effectively smoothes the EOS in the transition region from a first-order phase transition into a smooth crossover [4], restoring the monotonic decrease of $v_{2}(b) / \varepsilon(b)$ seen also in Figs. 3(a) and 3(c).

Our key point, however, is made by the right panels in Figs. 3 and 4. In these we show the swapped ratios that one would obtain if nature chose fKLN initial conditions but we as physicists scaled the corresponding experimentally measured elliptic flow $v_{2}^{\mathrm{fKLN}}$ incorrectly by dividing by the initial source eccentricity from the Glauber model (top right panels), or vice versa (bottom right panels). The qualitatively different shapes of the curves in the top right and bottom right panels of Figs. 3 and 4 are a direct reflection of the strong centrality dependence of the $\varepsilon_{\mathrm{fKLN}} / \varepsilon_{\mathrm{G}}$ eccentricity ratio shown in the inset of Fig. 1. It causes the swapped ratio $v_{2}^{\mathrm{fKLN}} / \varepsilon_{\mathrm{G}}$ in the top right panel to drop much more steeply with increasing $b$ than either of the correctly scaled ratios, overcoming even 
the stiffening effects at large $b$ from SM-EOS Q in the ideal fluid case. More importantly, it causes the other swapped ratio $v_{2}^{\mathrm{G}} / \varepsilon_{\mathrm{fKLN}}$ to increase with $b$ from central to midperipheral ( $b \sim 6-8 \mathrm{fm}$ ) collisions. This increase holds even for EOS I, over the entire range of $\eta / s$ explored here, but it is further strengthened at RHIC energies when using SM-EOS Q which effectively stiffens as $b$ increases. With a more realistic EOS that exploits the latest lattice QCD data and replaces the first order transition by a smooth crossover [20] we expect a $b$ dependence of $v_{2} / \varepsilon$ that interpolates between the shapes in Figs. 3 and 4.

Available data from $\mathrm{Au}+\mathrm{Au}$ and $\mathrm{Cu}+\mathrm{Cu}$ collisions at RHIC indicate that $v_{2} / \varepsilon$ falls monotonically from central to peripheral collisions, irrespective of whether one uses $\varepsilon_{\mathrm{fKLN}}$ or $\varepsilon_{\mathrm{G}}$ to scale the measured elliptic flow $v_{2}$ [14,21-23]. Figures 3(d) and 4(d) then appear to exclude the possibility that the measured $v_{2}$ arises from flow driven by Glauber initial conditions. Furthermore, Figs. 4(a) and 4(c) exclude the possibility that the fireball medium behaves as an inviscid ideal fluid. Our main result is that the comparison of our calculations with existing data on the centrality dependence of the eccentricity-scaled elliptic flow eliminates the optical Glauber model as a viable source of the initial source eccentricity. This is a qualitative finding based on the positive sign of the slopes of the curves shown in Fig. 4(d), which disagrees with the measured [21-23] negative slope. It does not depend on finer details of the data and thus appears to be robust. Our analysis further suggests that collective flow in heavy-ion collisions at RHIC may be driven by fKLN-like initial conditions and that the fireball evolves as a viscous fluid.

Extracting the precise value of its viscosity requires a quantitative study of elliptic flow data, including a description of the nonequilibrium stages preceding and following the hydrodynamic one and supplemented by additional observables such as the azimuthal dependence of jet suppression [24]. Furthermore, to obtain the viscosity of the interesting QGP stage one must subtract from the measured elliptic flow contributions arising from the highly viscous late hadronization and freezeout stages where the deviations from local equilibrium become large [25] and both shear and bulk viscosity are important (see Ref. [15] and references therein). This is not the goal of the present work. We here provide the basis for a binary decision tree that allows to distinguish between the optical Glauber and fKLN initialization models, thereby eliminating the largest prevailing uncertainty from such an extraction and thus clearing the path to eventual success of such an effort.

In closing, we point out two issues that require further study: (i) Our analysis assumes that the fireball medium has constant $\eta / s$ independent of collision centrality (and thus of temperature). Theory strongly suggests that $\eta / s$ increases during hadronization and is significantly larger in the late hadronic than in the early QGP phase. If the effective $\eta / s$ (averaged over the expansion history) increases dramatically with $b$, because of the larger role of the hadron phase in peripheral collisions, it may turn the rise of $\left(v_{2} / \varepsilon\right)(b)$ in Fig. 4(d) into a monotonic decrease, similar to the one seen in experiment. For this to happen, the effective $\eta / s$ would have to increase from, say, $1 / 4 \pi$ in central collisions to above $3 / 4 \pi$ at $b \sim 7 \mathrm{fm}$. Though we believe this to be unlikely, only an explicit calculation with a realistic model for the temperature dependence of $\eta / s$ (and also including bulk viscosity) can definitively rule out this possibility. (ii) We here used initial source eccentricities computed from the so-called "optical" versions of the Glauber and fKLN models. These do not include the event-by-event fluctuations of the initial source eccentricity that occur in heavy-ion collision experiments. If the ensemble-averaged effective eccentricities that include these event-by-event fluctuations exhibit a centrality dependence different than shown in Fig. 1 (in particular for the ratio between the Glauber and fKLN eccentricities in the inset of Fig. 1), our conclusions could be affected [26,27]. A study of fluctuating initial conditions is therefore needed and planned for the future.

U.H. acknowledges fruitful discussions with B. Jacak that triggered this study. We thank A. Dumitru and Y. Nara for providing the fKLN code $[12,18]$ and W. Horowitz, A. Majumder, B. Müller, and J. Nagle for useful comments. This work was supported by the US Department of Energy under Contract DE-FG02-01ER41190. J.S.M. gratefully acknowledges support by the Edward R. Grilly summer research program and by the undergraduate research program of Ohio State University.
[1] U. Heinz and S. M. H. Wong, Phys. Rev. C 66, 014907 (2002).

[2] D. Teaney, Phys. Rev. C 68, 034913 (2003).

[3] P. Romatschke and U. Romatschke, Phys. Rev. Lett. 99, 172301 (2007); H. Song and U. Heinz, Phys. Lett. B658, 279 (2008); K. Dusling and D. Teaney, Phys. Rev. C 77, 034905 (2008).

[4] H. Song and U. Heinz, Phys. Rev. C 77, 064901 (2008).

[5] M. Luzum and P. Romatschke, Phys. Rev. C 78, 034915 (2008); [Erratum-ibid. 79, 039903 (2009)].

[6] H. Song and U. Heinz, J. Phys. G 36, 064033 (2009).

[7] L. P. Csernai, J. I. Kapusta, and L. D. McLerran, Phys. Rev. Lett. 97, 152303 (2006).

[8] P. K. Kovtun, D. T. Son, and A. O. Starinets, Phys. Rev. Lett. 94, 111601 (2005); G. Policastro, D. T. Son, and A. O. Starinets, ibid. 87, 081601 (2001).

[9] T. Schäfer, Phys. Rev. A 76, 063618 (2007).
[10] P. Danielewicz and M. Gyulassy, Phys. Rev. D 31, 53 (1985).

[11] T. Hirano, U. Heinz, D. Kharzeev, R. Lacey, and Y. Nara, Phys. Lett. B636, 299 (2006); A. Kuhlman, U. Heinz, and Y. V. Kovchegov, ibid. B638, 171 (2006).

[12] A. Adil, H.-J. Drescher, A. Dumitru, A. Hayashigaki, and Y. Nara, Phys. Rev. C 74, 044905 (2006); H.-J. Drescher and Y. Nara, ibid. 75, 034905 (2007).

[13] H. Song and U. Heinz, Phys. Rev. C 78, 024902 (2008).

[14] M. Luzum and P. Romatschke, arXiv:0901.4588 [nucl-th].

[15] H. Song and U. Heinz, Phys. Rev. C (in press), arXiv:0909.1549 [nucl-th].

[16] P. F. Kolb, U. Heinz, P. Huovinen, K. J. Eskola, and K. Tuominen, Nucl. Phys. A696, 197 (2001).

[17] D. Kharzeev, E. Levin, and M. Nardi, Phys. Rev. C 71, 054903 (2005). 
[18] The code can be downloaded from https://wiki.bnl.gov/ TECHQM/index.php/CGC_initial_conditions_for_nuclear_ collisions.

[19] P. F. Kolb, J. Sollfrank, and U. Heinz, Phys. Lett. B459, 667 (1999); Phys. Rev. C 62, 054909 (2000).

[20] A. Bazavov et al., Phys. Rev. D 80, 014504 (2009).

[21] B. Alver et al. (PHOBOS Collaboration), Phys. Rev. Lett. 98, 242302 (2007).

[22] H. J. Drescher, A. Dumitru, C. Gombeaud, and J. Y. Ollitrault, Phys. Rev. C 76, 024905 (2007).
[23] H. Masui, J. Y. Ollitrault, R. Snellings, and A. Tang, Nucl. Phys. A830, 463c (2009).

[24] A. Adil, M. Gyulassy, and T. Hirano, Phys. Rev. D 73, 074006 (2006)

[25] C. Anderlik, Z. I. Lazar, V. K. Magas, L. P. Csernai, H. Stöcker, and W. Greiner, Phys. Rev. C 59, 388 (1999).

[26] J. L. Nagle, P. Steinberg, and W. A. Zajc, arXiv:0908.3684 [nucl-th].

[27] J. Nagle and L. A. Linden Levi (private communication). 